\title{
Experimental validation of gallium production and isotope-dependent positron range correction in PET
}

\author{
L.M. Fraile ${ }^{\text {a,** }}$, J.L. Herraiz ${ }^{\text {a }}$, J.M. Udías ${ }^{\text {a }}$, J. Cal-González ${ }^{a, 1}$, P.M.G. Corzo ${ }^{a, 2}$, S. Españaa ${ }^{a, 3}$, \\ E. Herranz ${ }^{\mathrm{a}, 4}$, M. Pérez-Liva ${ }^{a}$, E. Picado ${ }^{\mathrm{a}, 5}$, E. Vicente ${ }^{\mathrm{a}, 6}$, A. Muñoz-Martín $^{\mathrm{b}}$, J.J. Vaquero ${ }^{\mathrm{c}}$ \\ ${ }^{a}$ Grupo de Física Nuclear, Dpto. Física Atómica, Molecular y Nuclear, Universidad Complutense de Madrid, Spain \\ ${ }^{\mathrm{b}}$ Centro de Microanálisis de Materiales, Universidad Autónoma de Madrid, E-28049 Madrid, Spain

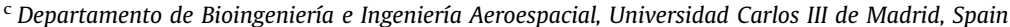

\section{A R T I C L E I N F O}

\section{Article history:}

Received 4 November 2015

Received in revised form

24 December 2015

Accepted 2 January 2016

Available online 22 January 2016

Keywords:

Radioisotope production

Positron emission tomography

Positron range

${ }^{66} \mathrm{Ga}$

${ }^{68} \mathrm{Ga}$

\begin{abstract}
A B S T R A C T
Positron range (PR) is one of the important factors that limit the spatial resolution of positron emission tomography (PET) preclinical images. Its blurring effect can be corrected to a large extent if the appropriate method is used during the image reconstruction. Nevertheless, this correction requires an accurate modelling of the PR for the particular radionuclide and materials in the sample under study. In this work we investigate PET imaging with ${ }^{68} \mathrm{Ga}$ and ${ }^{66} \mathrm{Ga}$ radioisotopes, which have a large PR and are being used in many preclinical and clinical PET studies. We produced a ${ }^{68} \mathrm{Ga}$ and ${ }^{66} \mathrm{Ga}$ phantom on a natural zinc target through $(\mathrm{p}, \mathrm{n})$ reactions using the $9-\mathrm{MeV}$ proton beam delivered by the 5-MV CMAM tandetron accelerator. The phantom was imaged in an ARGUS small animal PET/CT scanner and reconstructed with a fully 3D iterative algorithm, with and without PR corrections. The reconstructed images at different time frames show significant improvement in spatial resolution when the appropriate PR is applied for each frame, by taking into account the relative amount of each isotope in the sample. With these results we validate our previously proposed PR correction method for isotopes with large PR. Additionally, we explore the feasibility of PET imaging with ${ }^{68} \mathrm{Ga}$ and ${ }^{66} \mathrm{Ga}$ radioisotopes in proton therapy.
\end{abstract}

(c) 2016 Elsevier B.V. All rights reserved.

\section{Introduction}

One of the main limitations to the spatial resolution achievable in $3 \mathrm{D}$ positron emission tomography (PET) arises from the range of positrons in tissue [1]. Positron range (PR) makes the distribution of the emission points of the $511 \mathrm{keV}$ annihilation gamma rays different than those of the positron emissions. If it is not well modelled and corrected for, it may lead to a sizeable blurring in the reconstructed PET images. While the spatial resolution with state-of-the-art small animal PET scanners such as the ARGUS [2] is of the order of $1 \mathrm{~mm}$, the mean positron range (PR) of some commonly used isotopes is significantly larger. For instance, some radionuclides of interest such as ${ }^{68} \mathrm{Ga}$ or ${ }^{82} \mathrm{Rb}$ have large positron

\footnotetext{
* Corresponding author. Tel.: + 3491394 4784; fax: + 34913945193.

E-mail address: lmfraile@ucm.es (L.M. Fraile).

${ }^{1}$ Present address: Medical University of Vienna, Vienna, Austria.

2 Present address: Blue Telecom Consulting, 28037 Madrid, Spain.

3 Present address: Centro Nacional de Investigaciones Cardiovasculares, 28029 Madrid, Spain.

${ }^{4}$ Present address: Massachusetts General Hospital and Harvard Medical School, Boston, MA, USA.

${ }^{5}$ Present address: Universidad Nacional, Heredia 86-3000, Costa Rica.

${ }^{6}$ Present address: Johns Hopkins University, Baltimore, MD, USA.
}

ranges on the order of $2-5 \mathrm{~mm}$ [3], which makes their use in preclinical PET imaging challenging. On the other hand significant differences of positron range values can be found in the literature and experimental measurements are clearly lacking, as discussed in [3].

Several methods have been introduced to avoid the blurring of the images $[4,5]$ and different PR corrections have been proposed. In a previous work [6] we have showed that one can take into account the material-dependent positron range by modelling its effects during 3D OSEM reconstruction procedure [7], once the properties of the local media are obtained from a CT image. In this approach the quality of images significantly improves with PR correction, rendering large positron range isotopes such as ${ }^{68} \mathrm{Ga}$ meaningful [6].

An experimental validation of this procedure with several PET isotopes with significantly different positron ranges, such as ${ }^{68} \mathrm{Ga}$ and ${ }^{66} \mathrm{Ga}$ (see Table 1 ) is still needed. This can be achieved with reduced systematic uncertainties if both isotopes are simultaneously produced in sufficient amounts at the same location, as proposed in this work.

The study of the production of Ga isotopes using proton beams has also interest for its potential medical applications, as they could play a role in non-invasive monitoring of proton radiotherapy [8] 
Table 1

Main properties of the radionuclides ${ }^{68} \mathrm{Ga}$ and ${ }^{66} \mathrm{Ga}$. Data taken from [13] (b.r.= branching ratio).

\begin{tabular}{lll}
\hline Isotope & ${ }^{68} \mathrm{Ga}$ & ${ }^{66} \mathrm{Ga}$ \\
\hline$T_{1 / 2}(\mathrm{~min})$ & $67.71(9)$ & $569(2)$ \\
Stable daughter & ${ }^{68} \mathrm{Zn}$ & ${ }^{66} \mathrm{Zn}$ \\
$\beta^{+}$decay b.r. & $89 \%$ & $57 \%$ \\
Mean $E_{\beta+}(\mathrm{keV}) / Q_{\beta+}(\mathrm{keV}) /[$ b.r.] & $353 / 822 /[1.2 \%]$ & $397 / 924 /[4 \%]$ \\
& $836 / 1899 /$ & $1905 / 4153 /$ \\
& {$[87.9 \%]$} & {$[50 \%]$} \\
\hline
\end{tabular}

via PET. Contrary to ${ }^{11} \mathrm{C}$ and ${ }^{15} \mathrm{O}$, which are produced by nuclear interactions along the proton beam path with a higher energy threshold (16.6 MeV for ${ }^{11} \mathrm{C}$ and $20.3 \mathrm{MeV}$ for ${ }^{15} \mathrm{O}$ ), ${ }^{66} \mathrm{Ga}$ and ${ }^{68} \mathrm{Ga}$ metal isotopes are produced at lower energies (see Fig. 1). This may allow a better measurement of the dose distribution deposited by the protons at the end of their trajectory, where they have a low energy and deposit the maximum amount of dose.

Zinc in the form of seeds or nanoparticles could be used as a contrast agent [9] for the target volume, and its distribution localized with a magnetic resonance (MR) acquisition. The gallium isotopes produced during the proton therapy treatment from the $\mathrm{Zn}(\mathrm{p}, \mathrm{n}) \mathrm{Ga}$ reactions $[10,11]$ in the zinc material, may then be measured in a PET scanner after the irradiation. The combination of the information provided by the MR and PET images, together with an accurate knowledge of the cross-section of these reactions, would allow a precise measurement of the dose deposited by the low-energy protons.

In this work we assessed the viability of production gallium PET isotopes and 3D image reconstruction with positron range corrections. We used a low-energy proton beam provided by the CMAM [14] facility impinging on a natural Zn target to produce the gallium isotopes of interest. This procedure is similar to what was used by Sattari et al. [15], but in our case with the beam we depict a Derenzo-like pattern on the target and we perform PET imaging in a small animal PET scanner. We correct for isotope-dependent positron range by modelling its effects during 3D OSEM reconstruction [7], taking into account the dominant radionuclide present in the sample.

\section{Material and methods}

\subsection{Isotope production}

The CMAM Cockcroft-Walton tandetron accelerator delivers proton beams of up to $10 \mathrm{MeV}$ with intensities of $3 \mu \mathrm{A}$ [14]. In this experiment we have used a proton energy of $9 \mathrm{MeV}$ in order to maintain a good current stability. This energy matches the proton energy at the distal edge of typical proton therapy beams [8]. The beam intensity has been chosen as a compromise between sizable reaction cross-sections and manageable activities (see below). A high-purity (99.99\%) natural zinc target $\left(\rho=7.13 \mathrm{~g} / \mathrm{cm}^{3}\right)$ has been used in this experiment, with natural abundances of $27.7 \%{ }^{66} \mathrm{Zn}$ and $18.4 \%{ }^{68} \mathrm{Zn}$. The production of our gallium isotopes of interest, ${ }^{66,68} \mathrm{Ga}$, occurs via $(\mathrm{p}, \mathrm{n})$ reactions, along with the short-lived $\beta^{+}$ emitter ${ }^{64} \mathrm{Ga}$ and also ${ }^{67} \mathrm{Ga}$, which decays entirely by EC with $T_{1}$ / ${ }_{2}=3.2617(3)$ days and provides suitable decay gamma radiation for monitoring purposes. The $(\mathrm{p}, \mathrm{n})$ reaction products induced by the $9 \mathrm{MeV}$ proton beam on natural $\mathrm{Zn}$ are compiled in Table 2 . The $9 \mathrm{MeV}$ beam was completely stopped in the first hundreds of microns of the $\mathrm{Zn}$ foil.

The activation was performed in the standard multipurpose beam line at CMAM (see Fig. 2), which includes an experimental chamber with a 4-axes programmable goniometer, with sufficient

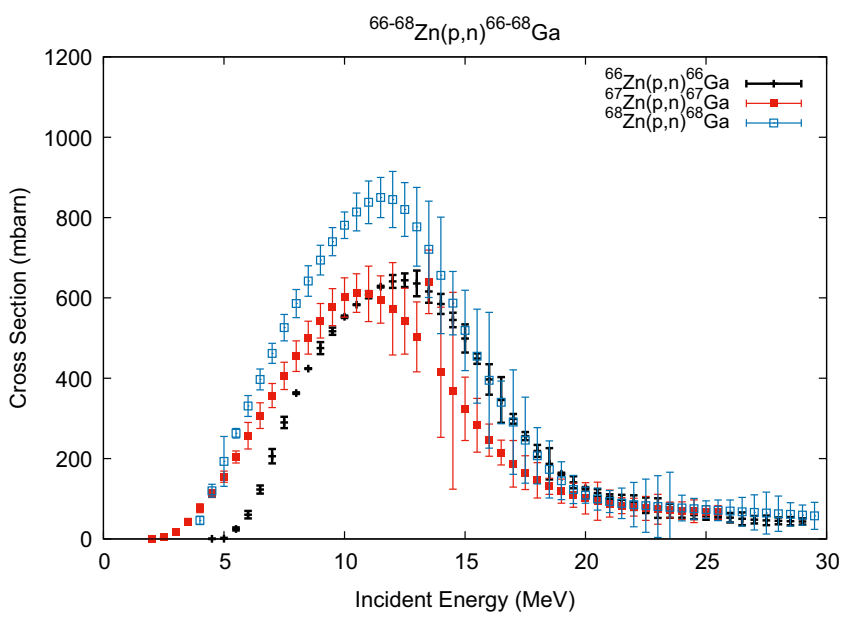

Fig. 1. Total cross-sections as a function of energy for the ${ }^{66} \mathrm{Zn}(\mathrm{p}, \mathrm{n}){ }^{66} \mathrm{Ga},{ }^{67} \mathrm{Zn}(\mathrm{p}, \mathrm{n}){ }^{67} \mathrm{Ga}$ and ${ }^{68} \mathrm{Zn}(\mathrm{p}, \mathrm{n}){ }^{68} \mathrm{Ga}$ reactions [12].

precision and speed in its axial and radial degrees of freedom for our purpose.

The proton beam was used to irradiate the natural zinc target foil and depict a pattern as presented in Fig. 3. This pattern is inspired by a quality control Derenzo phantom, commonly used in nuclear medicine imaging, which consists of a cylinder divided in six sectors, each filled with rods of different diameters with centre-to-centre separation equal to twice their diameter. The rods contain a solution of the desired activity. In our case we activated square areas fitting the beam collimation, with increasing sides of 1, 2 and $3 \mathrm{~mm}$ (see Fig. 3).

The beam was collimated to achieve uniform activation in the spots and to minimize any border effect. The irradiation times and beam intensity were adjusted to attain the same charge per unit area of the order of $28 \mathrm{nC} / \mathrm{mm}^{2}$, which leads to similar activity per unit surface in each spot. The dimensions, currents and irradiation times per spot are compiled in Table 3.

\subsection{Activity of the ${ }^{66} \mathrm{Ga}$ and ${ }^{68} \mathrm{Ga}$ isotopes}

The production of the gallium isotopes is governed by the cross-sections plotted in Fig. 4. From these values the thick target yield $Y(E)$ can be obtained from the expression:

$Y(E)=\rho_{A} \int_{0}^{E} \sigma\left(E^{\prime}\right)\left(\frac{d E^{\prime}}{d x}\right)^{-1} d E^{\prime}$

where $\rho_{A}$ is the target atomic density expressed in atoms per $\mathrm{cm}^{3}$, $E$ is the proton energy at the entrance of the material $(9.0 \mathrm{MeV}$ in this case), $\sigma\left(E^{\prime}\right)$ is the cross-section plotted in Fig. 3 and $d E^{\prime} / d x$ is the (energy dependent) energy loss of protons in $\mathrm{Zn}$, as shown in Fig. 4. A target thickness larger than the range of the protons at this energy is explicitly assumed in (1), which is the case in our experiment.

The expected activity and the count rate can be obtained by calculating the amount of radionuclides produced per unit time $(\Gamma)$ in a thick target by a beam of protons of energy $E$ as

$\Gamma=\phi Y(E)$,

where $\phi$ is the proton flux $\left(\mathrm{s}^{-1}\right)$ for the selected beam current. The thick target yield $Y(E)$ can be calculated by considering successive thin slices of target material. Alternatively, given the smooth dependence of the cross-section with the penetration depth (see plot in Fig. 4), which takes into account the trend of the energy loss, the amount of radionuclides produced per unit time can be 
Table 2

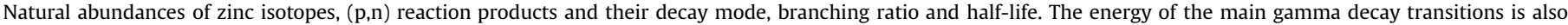
listed.

\begin{tabular}{|c|c|c|c|c|c|c|}
\hline Natural zinc isotopes & Abundance (\%) & $(\mathrm{p}, \mathrm{n})$ reaction product & $T_{1 / 2}$ & Main decay mode & Branching ratio & $E(\mathrm{keV})$ of main $\gamma$ decay transitions \\
\hline${ }^{64} \mathrm{Zn}$ & 49.2 & ${ }^{64} \mathrm{Ga}$ & $2.627(12) \min$ & $\beta^{+}$ & 97.5 & $511.0,991.5,807.5,3365.9$ \\
\hline${ }^{66} \mathrm{Zn}$ & 27.7 & ${ }^{66} \mathrm{Ga}$ & $9.49(3) \mathrm{h}$ & $\boldsymbol{\beta}^{+}$ & $\mathbf{5 7 . 0}$ & $511.0,1039.2,2751.8,833.5$ \\
\hline${ }^{67} \mathrm{Zn}$ & 4.0 & ${ }^{67} \mathrm{Ga}$ & $3.2617(5) \mathrm{d}$ & $\mathrm{EC}$ & 100.0 & $93.3,184.6,300.2$ \\
\hline${ }^{68} \mathrm{Zn}$ & 18.5 & ${ }^{68} \mathbf{G a}$ & $67.71(9) \mathrm{min}$ & $\boldsymbol{\beta}^{+}$ & 88.9 & $511.0,1077.3$ \\
\hline${ }^{70} \mathrm{Zn}$ & 0.6 & ${ }^{70} \mathrm{Ga}$ & $21.14(9) \mathrm{min}$ & $\beta^{-}$ & 99.6 & (1039.2), (176.2) \\
\hline
\end{tabular}

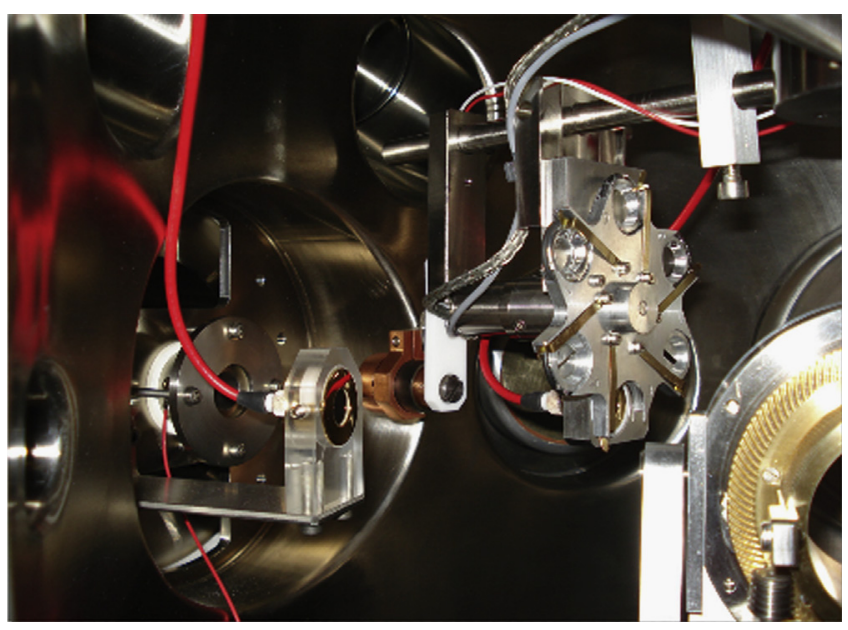

Fig. 2. The experimental chamber at the standard beam line at CMAM.

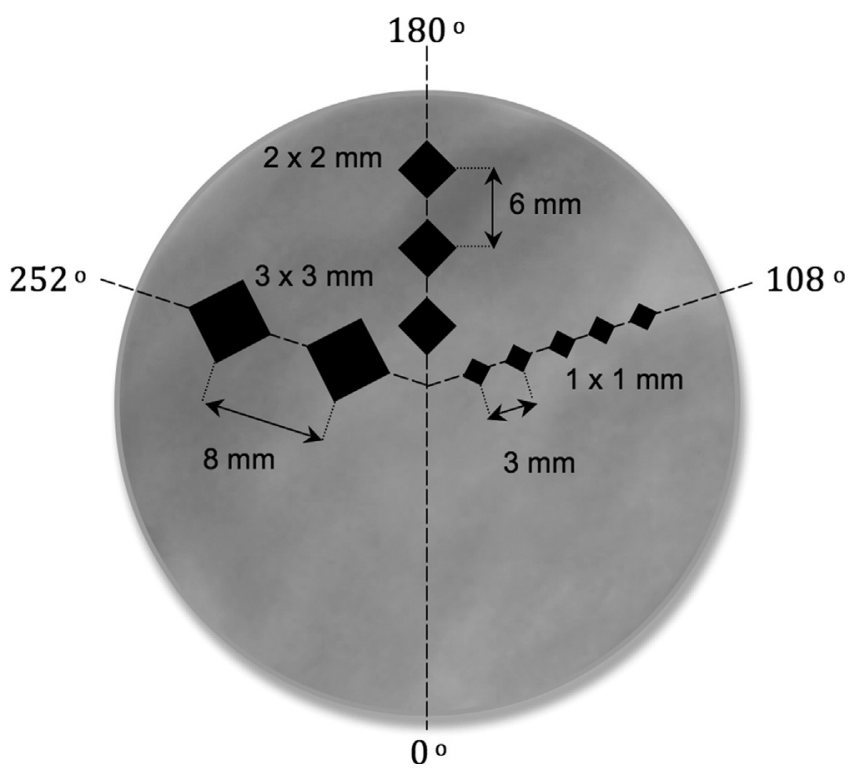

Fig. 3. Sketch of the Derenzo-like pattern activated with the $9 \mathrm{MeV}$ proton beam on the $\mathrm{Zn}$ target.

estimated using

$\Gamma \equiv \phi f \rho_{A} r \hat{\sigma}$

where $\rho_{A}$ is the atomic density of natural $\mathrm{Zn}, f$ the fraction of the isotope of interest in the target material, $r$ is the penetration depth of the protons in the material for which the cross-section is non-negligible, and $\hat{\sigma}$ the average cross-section for the given penetration depth.

In our case we calculated the amount of ${ }^{66,68} \mathrm{Ga}$ produced per unit time using discrete energy steps of $0.5 \mathrm{MeV}$ from $9 \mathrm{MeV}$ down
Table 3

Irradiation times and intensities for each of the spots.

\begin{tabular}{lllll}
\hline$\theta(\mathrm{deg})$ & $r(\mathrm{~mm})$ & Size $(\mathrm{mm})$ & Time $(\mathrm{s})$ & $I(\mathrm{nA})$ \\
\hline 252 & 6,14 & $3 \times 3$ & $2 \times 40$ & 6.3 \\
180 & $5,11,17$ & $2 \times 2$ & $3 \times 36$ & 3.1 \\
108 & $5,8,11,14,17$ & $1 \times 1$ & $5 \times 39$ & 0.78 \\
\hline
\end{tabular}

to the energy where the cross-section vanishes (see Fig. 4). The amount of ${ }^{67} \mathrm{Ga}$, arising from the ${ }^{67} \mathrm{Zn}(\mathrm{p}, \mathrm{n}){ }^{67} \mathrm{Ga}$ reaction, which serves as activity monitor, has been calculated in the same manner. The penetration in the $\mathrm{Zn}$ foil was obtained from the stopping power factor computed with SRIM [16].

After the PET measurements described in next section were performed, the decay of ${ }^{67} \mathrm{Ga}$ in the foil was also used to monitor the activity in the target and to verify the production crosssections. For that purpose high-resolution gamma spectrometry was performed with a LOAX-type HPGe detector [17], whose absolute efficiency had been previously calibrated.

Measurements of ${ }^{67} \mathrm{Ga}$ were performed 8 days after the irradiation, once ${ }^{66} \mathrm{Ga}$ (and other shorter-lived isotopes such as ${ }^{68} \mathrm{Ga}$ ), had almost completely disappeared. Several individual measurements over a period of 5 half-lives of ${ }^{67} \mathrm{Ga}$, each lasting for $20,000 \mathrm{~s}$, were done. An example is shown in the gamma spectrum of Fig. 5, where the most intense ${ }^{67} \mathrm{Ga}$ gamma decay peaks (see Table 2) can be clearly identified. Some of the gamma rays from the decay of ${ }^{66} \mathrm{Ga}$, including the $511-\mathrm{keV}$ gamma, were weakly observed at higher energies. The activity of the ${ }^{67} \mathrm{Ga}$ gamma rays, diminishing with the ${ }^{67} \mathrm{Ga}$ decay constant, was studied in order to obtain the original activity after the irradiation. The result was consistent with the estimate of $0.25 \mathrm{kBq}$ discussed in Section 3.

\subsection{PET image reconstruction with isotope-dependent positron range correction}

After the irradiation, the $\mathrm{Zn}$ foil with the Ga Derenzo-like pattern was submerged in water and measured in the ARGUS small animal PET/CT scanner [2]. The Ga activity was concentrated on a superficial thin slice of less than $250 \mu \mathrm{m}$ on the $\mathrm{Zn}$ target. The measurements started $2.25 \mathrm{~h}$ after the end of the bombardment (EOB) of the foil, and 27 frames (1200 s each) were acquired. Frames 1, 7 and 25 of the acquisition were chosen for image reconstruction, since they are representative of the different admixtures of activities of the Ga isotopes. An energy window was defined from $400 \mathrm{keV}$ to $700 \mathrm{keV}$, which minimizes the contribution from the intrinsic activity of the LYSO crystals.

The images were reconstructed with the iterative reconstruction code FIRST [7,18], with and without PR correction [6]. The algorithm included the one-step-late maximum-a-posteriori (OSLMAP) regularization described in [19] with $\beta=0.1$. Forty iterations of the algorithm were used in all cases. The reconstructed images consisted of $175 \times 175 \times 61$ voxels of $0.388 \times 0.388 \times 0.775 \mathrm{~mm}^{3}$.

As shown in our previous works $[6,20]$ PR correction can be introduced into the iterative image reconstruction using the PR 


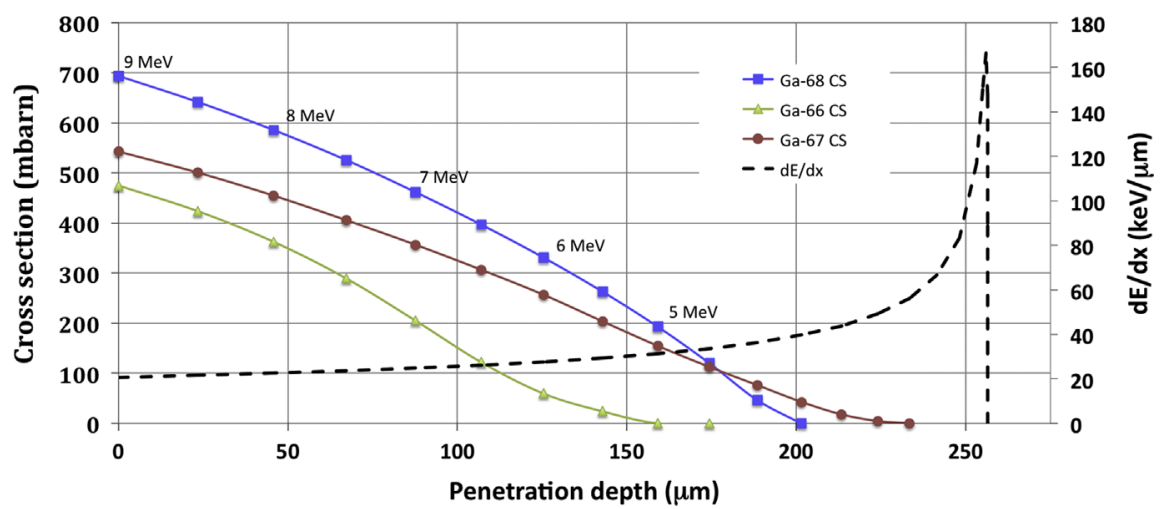

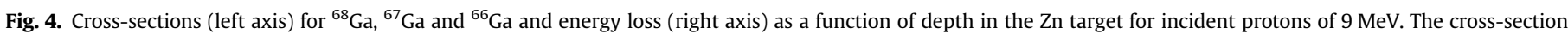
values are plotted in decreasing steps of $0.5 \mathrm{MeV}$, starting at $9 \mathrm{MeV}$.

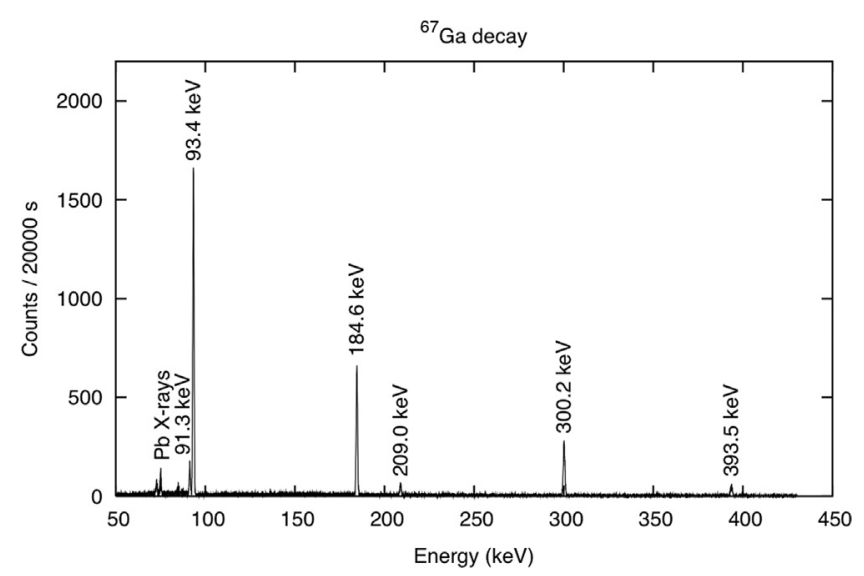

Fig. 5. Low energy gamma rays from the remaining activity in the activated $\mathrm{Zn}$ foil, obtained 8 days after irradiation with a HPGe detector. They correspond mainly to the ${ }^{67}$ Ga decay (labelled lines).

distribution obtained from Monte Carlo simulations [3] as an additional blurring applied to the object. In this case, the system response model simulated with PeneloPET [21] should not incorporate the PR effects, but must include all other physical effects like non-colinearity or inter-crystal scatter.

Following a similar approach as in $[6,20]$ the PR correction is only applied in the forward projection step. Using it also in the backward projection makes the algorithm to converge more slowly without improving the images [6]. The PR-corrected OSEM (PR-OSEM) iterative algorithm is:

$\tilde{x}_{j}=x_{j} \frac{\sum_{i} A_{i j}\left(\frac{y_{i}}{\sum_{j} A_{i j} \tilde{x}_{j}}\right)}{\sum_{i} A_{i j}}$,

where $\tilde{x}_{j}$ is the image blurred by PR that is forward projected. $\tilde{x}_{j}$ is obtained by the convolution of the initial image $x_{j}$ with a kernel corresponding to the annihilation Point Spread Function (aPSF):

$\tilde{x}_{j}=x_{j} \otimes a P S F$.

The $a P S F$ is the 3-dimensional range profile corresponding to the isotope source and material present at voxel $j$. In this work we used a PR kernel of $25 \times 25 \times 13$ voxels of $0.388 \times 0.388 \times 0.775 \mathrm{~mm}^{3}$ that was sufficient to describe the PR distribution of the Ga isotopes in $\mathrm{Zn}$ and water. The different PR of ${ }^{68} \mathrm{Ga}$ and ${ }^{66} \mathrm{Ga}$ in $\mathrm{Zn}$ and water are shown in Fig. 6.

The Ga isotopes were formed on the surface of the disc, as the maximum depth in which they were created by the proton beam is $0.2 \mathrm{~mm}$ (Fig. 4). Therefore, the material of the voxels of the image containing the activated Ga sources can be considered a mixture of

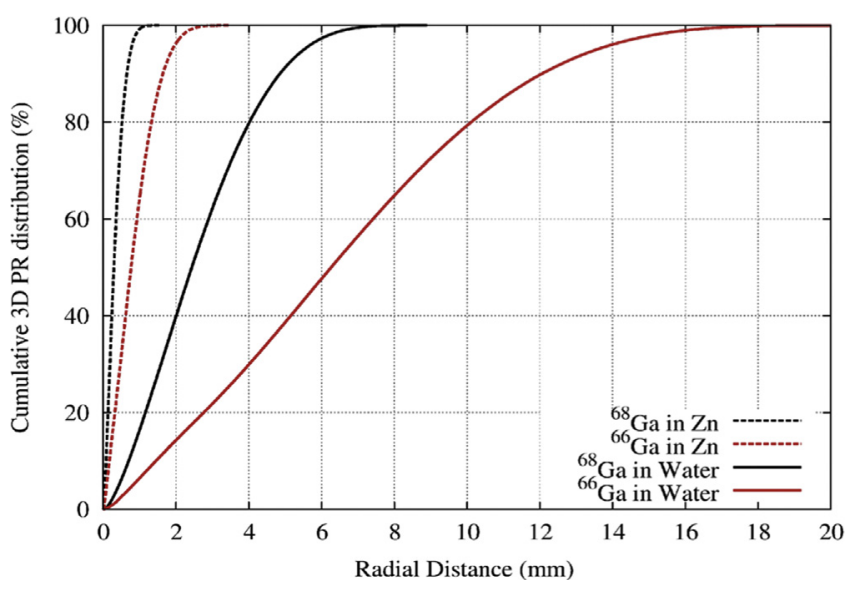

Fig. 6. Positron range cumulative 3 -dimensional distribution for ${ }^{66} \mathrm{Ga}$ and ${ }^{68} \mathrm{Ga}$ in water and $\mathrm{Zn}$.

Table 4

Mean PR obtained in this work with PeneloPET for ${ }^{66} \mathrm{Ga}$ and ${ }^{68} \mathrm{Ga}$ in water and $\mathrm{Zn}$. Results from literature are presented for comparison.

\begin{tabular}{lllll}
\hline Isotope & Material & \multicolumn{2}{l}{$R_{\text {mean }}(\mathrm{mm})$} & \\
\cline { 3 - 5 } & & $\begin{array}{l}\text { PeneloPET (this } \\
\text { work) }\end{array}$ & $\begin{array}{l}\text { Other } \\
\text { simulations }\end{array}$ & Experiment \\
\hline${ }^{68} \mathrm{Ga}$ & Water & 2.45 & $\begin{array}{l}3.56[22] / 2.9 \\
{[23]}\end{array}$ & $\begin{array}{l}2.8[24] / 1.975 \\
{[25]}\end{array}$ \\
& Zn & 0.30 & - & - \\
& & & $6.13[26]$ & - \\
${ }^{66} \mathrm{Ga}$ & Water & 6.27 & - & - \\
& $\mathrm{Zn}$ & 0.77 & &
\end{tabular}

$\mathrm{Zn}$ and water. We used the approximation of considering a homogeneous PR in (2) with a distribution obtained as the mean of the distributions in $\mathrm{Zn}$ and water.

In the acquisition there was a mixture of two PET isotopes in the sample. Therefore, we also had to use an admixture of their PR kernels to perform the correct PR correction. The distinctive halflives of both PET isotopes make this mixture to be different in each frame. In the initial frame the admixture contained $85 \%$ of ${ }^{68} \mathrm{Ga}$ and $15 \%$ of ${ }^{66} \mathrm{Ga}$ PR kernels. For the second reconstruction (frame 7), we combined $64 \%$ of ${ }^{68} \mathrm{Ga}$ and $36 \%$ of ${ }^{66} \mathrm{Ga}$, and in the last one (frame 25), we mixed $4 \%$ of ${ }^{68} \mathrm{Ga}$ and $96 \%$ of ${ }^{66} \mathrm{Ga}$.

\section{Results and discussion}

The calculated amount of radionuclides produced per unit time and $\mathrm{nA}$ of proton beam (i.e. per $\mathrm{nC}$ on target) was 

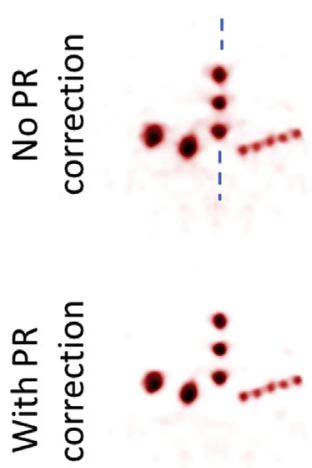

FRAME 1

${ }^{68} \mathrm{Ga}(85 \%)$

${ }^{66} \mathrm{Ga}(15 \%)$
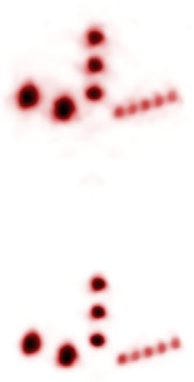

FRAME 7

${ }^{68} \mathrm{Ga}(64 \%)$

${ }^{66} \mathrm{Ga}(36 \%)$
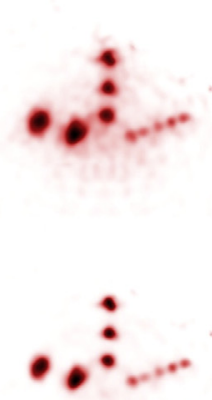

. 0

FRAME 25

${ }^{68} \mathrm{Ga}(4 \%)$

${ }^{66} \mathrm{Ga}(96 \%)$

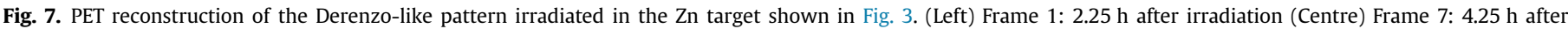

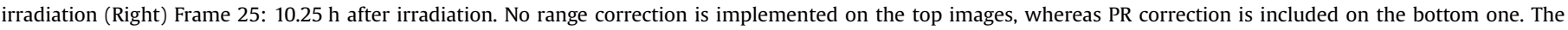
relative amount of the Ga isotopes contributing to the PET image is also shown. The profiles along the line shown across the 2 mm spots are plotted in Fig. 8.

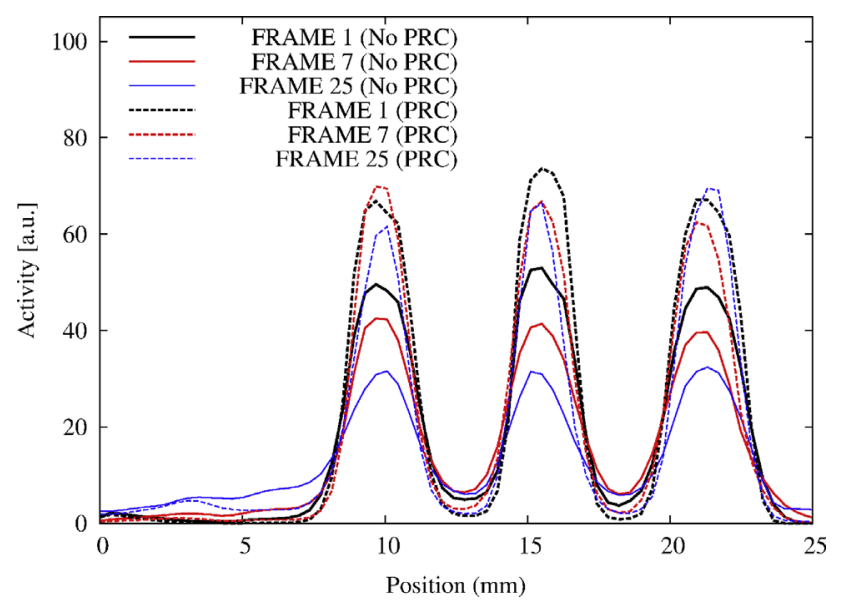

Fig. 8. Profiles through the $2 \mathrm{~mm}$ Ga sources for the frame 1 (mostly ${ }^{68} \mathrm{Ga}$ ), frame 7 (mixture), and frame 25 (mostly ${ }^{66} \mathrm{Ga}$ ) shown in Fig. 7, with PR correction (PRC) and without it (No PRC). By using the PRC, the peak to valley ratio is improved by a factor of 6 (frame 1), 5 (frame 7 ) and 6 (frame 25).

$\left({ }^{66} \mathrm{Ga}\right)=4.23 \times 10^{5} \mathrm{nC}^{-1},\left({ }^{67} \mathrm{Ga}\right)=1.04 \times 10^{5} \mathrm{nC}^{-1}$ and $\left({ }^{68} \mathrm{Ga}\right)=6.00 \times$ $10^{5} \mathrm{nC}^{-1}$. The estimated relative uncertainty based on the tabulated cross-section energy and on the approximation by Eq. (3) is of the order of $10 \%$. From the production rates and the isotope decay probabilities, the activities of the different isotopes in the foil at the EOB are calculated to be $\mathrm{A}\left({ }^{66} \mathrm{Ga}\right)=8.5 \mathrm{kBq}, \quad \mathrm{A}\left({ }^{67} \mathrm{Ga}\right)=0.25 \mathrm{kBq}$ and $\mathrm{A}\left({ }^{68} \mathrm{Ga}\right)=99 \mathrm{kBq}$. Therefore, the expected activities from the crosssection values when the PET imaging was started $(2.25 \mathrm{~h}$ after the EOB, see Section 2), was $7.2 \mathrm{kBq}$ of ${ }^{66} \mathrm{Ga}$ and $25.8 \mathrm{kBq}$ of ${ }^{68} \mathrm{Ga}$, yielding a total of $33.0 \mathrm{kBq}$, since ${ }^{67} \mathrm{Ga}$ is not a positron emitter. Therefore, using the branching ratios of $\beta^{+}$emission from Table 1 , the expected amount of positron emissions per second at the beginning of the PET acquisition was $27 \pm 3 \mathrm{kBq}$. An activimeter was used at that moment, $2.25 \mathrm{~h}$ after EOB, to experimentally measure the target $\beta^{+}$activity via $511-\mathrm{keV}$ photons, perfectly matching the expected ${ }^{66,68} \mathrm{Ga}$ isotope activity.

The cumulative 3-dimensional distributions of the PR of ${ }^{66} \mathrm{Ga}$ and ${ }^{68} \mathrm{Ga}$ in water and $\mathrm{Zn}$ obtained from the MC simulations are shown in Fig. 6. It shows the percentage of emitted positrons that are stopped in the material within a sphere of radius $r$. This kind of representation gives a better idea of the PR distribution than other proposed ones, like profiles through the 3D-distribution, or projections over specific angles [3]. Table 4 presents the calculated mean positron ranges for ${ }^{66} \mathrm{Ga}$ and ${ }^{68} \mathrm{Ga}$ in water and $\mathrm{Zn}$, obtained from the MC simulations. Results from other simulations or experimental data, when available, are also shown.

It can be seen in Fig. 6 that $95 \%$ of the positrons emitted by ${ }^{68} \mathrm{Ga}$ will annihilate within a sphere of $0.76 \mathrm{~mm}$ radius in $\mathrm{Zn}$ and $5.5 \mathrm{~mm}$ in water. On the other hand, ${ }^{66} \mathrm{Ga}$ emits positrons with much larger energy, and 95\% of them are stopped within $1.9 \mathrm{~mm}$ in $\mathrm{Zn}$ and $13.5 \mathrm{~mm}$ in water. The differences in the PR between isotopes and materials become apparent.

Fig. 7 shows the PET reconstruction of the Derenzo-like pattern irradiated in the $\mathrm{Zn}$ target of 3 acquisitions: $2.25,4.25$ and $10.25 \mathrm{~h}$ after EOB, with 20-, 40- and 180-min acquisition, respectively. The duration of the frames was chosen in order to have a similar amount of coincident events in each dataset. The reconstruction was performed without (top row) and with (bottom row) PR correction. It is important to note how the large positron range of ${ }^{66} \mathrm{Ga}$ creates not only an image with lower resolution, but also with significantly larger background.

Fig. 8 shows the profiles through these images along the $2 \mathrm{~mm}$ spots indicated by the line shown in the top left of Fig. 7. The curves have not been renormalized, so the different heights are compensated by the different width and background. The profiles show how the blurring and background caused by the PR can be cured with the appropriate PR correction.

This has a significant impact on the images obtained with isotopes that emit positrons with large initial energy such as ${ }^{66} \mathrm{Ga}$. The quantitative analysis of these images indicates that a resolution down to $1.5 \mathrm{~mm}$ can be obtained with ${ }^{66} \mathrm{Ga}$ with PR correction, versus $2.5 \mathrm{~mm}$ without it. The peak to valley ratio is greatly improved by a factor of more than 5 and the reconstructed apparent size and peak height of the activity profiles with different mixtures of isotopes is similar. With our approach, the resolution and peak to background ratio is recovered in the PR-corrected images for ${ }^{68} \mathrm{Ga}$ and ${ }^{66} \mathrm{Ga}$.

Additionally, we have explored the feasibility of PET imaging with the produced ${ }^{68} \mathrm{Ga}$ and ${ }^{66} \mathrm{Ga}$ radioisotopes in the context of proton therapy. Nanoparticles of high- $Z$ materials have been proposed as radiosensitizers aimed at enhancing radiation dose delivered to tumours [27]. In this work we verified that the generated $\beta^{+}$activity in the $\mathrm{Zn}$ target matches the expected values from the tabulated cross-sections within $10 \%$ uncertainty, in view of a possible use of $\mathrm{Zn}$ seeds for PET monitoring of proton therapy. Assuming a density of $\mathrm{Zn}$ ferrite nanoparticles of $10 \mathrm{mg}$ per gram of tissue [28], a proton beam of $140 \mathrm{MeV}$ will produce a ${ }^{68} \mathrm{Ga}$ 


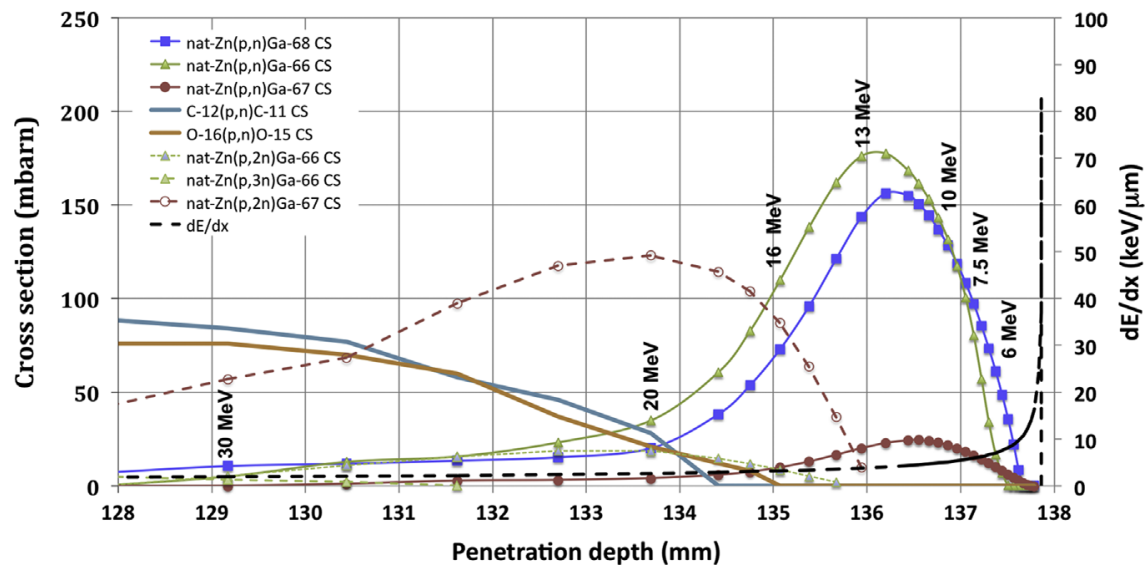

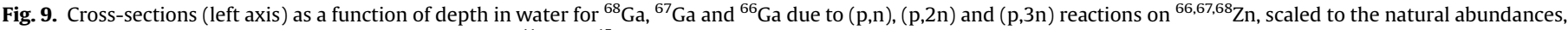

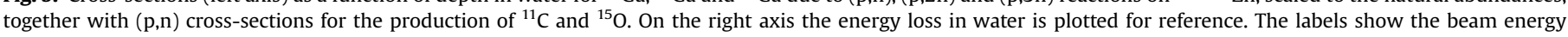
in $\mathrm{MeV}$.

activity of $4 \mathrm{~Bq}$ per $\mathrm{nC}$ of deposited charge (i.e. $1 \mathrm{nA}$ in $1 \mathrm{~s}$ ), and about $0.75 \mathrm{~Bq}$ of ${ }^{66} \mathrm{Ga}$. For the above estimate the stopping power of protons in water has been used, and a density of $1 \mathrm{~g} / \mathrm{cm}^{3}$ is assumed (see Fig. 9).

This activity is produced in the last $\sim 5 \mathrm{~mm}$ of the proton range before stopping, where virtually no other reaction channels are open, in particular the production of ${ }^{11} \mathrm{C}$ or ${ }^{15} \mathrm{O}$ in tissue. As a comparison, the activity production rates at the $\mathrm{EOB}$ for ${ }^{10,11} \mathrm{C}$ and ${ }^{15} \mathrm{O}$ are reported in [29] to be $109 \pm 12 \mathrm{~Bq} / \mathrm{s}$ for a $140 \mathrm{MeV} 16 \mathrm{pA}$ proton beam, over the whole range $(12.1 \mathrm{~cm})$ for the beam completely stopped in an acrylic glass (PMMA) phantom. Although this is higher than the EOB activity density from the activated $\mathrm{Zn}$, the Ga activity would be localized in few millimetres of tissue, and secondly the half-life of ${ }^{66} \mathrm{Ga}$ and ${ }^{68} \mathrm{Ga}$ is much larger than ${ }^{11} \mathrm{C}\left(T_{1 / 2}=20.39 \mathrm{~min}\right),{ }^{15} \mathrm{O}\left(T_{1 / 2}=2.037 \mathrm{~min}\right)$ and ${ }^{10} \mathrm{C}$ $\left(T_{1 / 2}=0.3218 \mathrm{~min}\right)$, making it possible for a PET acquired $2 \mathrm{~h}$ after EOB to mostly show the activity coming from the Ga isotopes. In any case, reaching $\mathrm{Ga}$ activities similar to those of $\mathrm{C}$ and $\mathrm{O}$ isotopes would require the use of macroscopic $\mathrm{Zn}$ seeds. It should be noted that the co-registration of the PET and MRI images of the zinc material would enable to quantify the density of $\mathrm{Zn}$ present in the target area.

\section{Conclusions}

In this work we have investigated an experimental method for producing phantoms for simultaneous PET imaging with gallium ${ }^{68} \mathrm{Ga}$ and ${ }^{66} \mathrm{Ga}$ isotopes. These radioisotopes can be simultaneously produced with a low energy proton beam, due to the low energy reaction threshold for production by $(p, n)$ reactions on a zinc target.

For this purpose an activation experiment has been performed at CMAM in Madrid. Short irradiation times at a low proton beam current have been used to depict a Derenzo-like pattern in a natural $\mathrm{Zn}$ foil, yielding a high-resolution activity-calibrated gallium phantom for PET imaging. The measured activity of the ${ }^{68} \mathrm{Ga}$ and ${ }^{66} \mathrm{Ga}$ beta emitters matches very well the estimates based on the evaluated $(p, n)$ reaction cross-sections and projected proton ranges in $\mathrm{Zn}$. Other reaction products did not disturb our purposes, either due to the half-life or to the absence of positron emission.

The irradiation leads to the simultaneous production of the two Ga isotopes with different positron ranges and half-lives, which can be employed to assess positron range corrected reconstruction methods. The reconstruction without using the appropriate PR distribution has a negative effect in the quality of reconstructed images (low resolution images with significant background), whereas the use of isotope-dependent PR correction yields a similar apparent size and peak height of activity profiles in acquisitions with different mixtures of isotopes. Therefore it can be successfully employed to recover the spatial resolution. In addition, these nuclides may play a role in off-line PET monitoring of proton radiotherapy due to their half-lives and appropriate $\beta$ end-point energy.

In conclusion, the approach proposed in this work improves significantly the quality of the reconstructed images, rendering PR corrected images for large PR isotopes, such as ${ }^{68} \mathrm{Ga}$ and ${ }^{66} \mathrm{Ga}$, practical and usable.

\section{Acknowledgements}

We acknowledge support from the Spanish MINECO through projects FPA2010-17142, FPA2013-41267-P, CSD-2007-00042 (CPAN), and the RTC-2015-3772-1 Grant. We also acknowledge support from Comunidad de Madrid via the TOPUS S2013/MIT3024 project.

\section{References}

[1] C.S. Levin, E.J. Hoffman, Physics in Medicine and Biology 44 (1999) 781.

[2] Y. Wang, et al., Journal of Nuclear Medicine 47 (2006) 1891

[3] J. Cal-González, et al., Physics in Medicine and Biology 58 (2013) 5127.

[4] S.E. Derenzo, IEEE Transactions on Nuclear Science NS33 (1) (1986) 565.

[5] B. Bai, R. Laforest, et al., IEEE Nuclear Science Symposium Conference Record (2004) 2501

[6] J. Cal-Gonzalez, et al., Nuclear Instruments and Methods in Physics Research A 648 (2011) S172.

[7] J.L. Herraiz, et al., Physics in Medicine and Biology 51 (2006) 4547.

[8] D. Schardt, et al., Reviews of Modern Physics 82 (2010) 383.

[9] J.H. Lee, et al., Nature Medicine 13 (2007) 95.

[10] C.J. Mathias, et al., Nuclear Medicine and Biology 30 (2003) 725.

[11] Ö. Ugur, et al., Nuclear Medicine and Biology 29 (2002) 147.

[12] EXFOR: Experimental nuclear reaction data 〈http://www.nndc.bnl.gov/exfor/ exfor00.htm>.

[13] Nuclear structure and decay data 〈http://www.nndc.bnl.gov/nudat2〉.

[14] Centro de Microanálisis de Materiales, CMAM, 〈http://www.cmam.uam.es $\rangle$.

[15] A. Sattari, et al. AJ31-5, Alasbimn Journal 8 (31) (2006).

[16] J.F. Ziegler, M.D. Ziegler, J.P. Biersack, Nuclear Instruments and Methods in Physics B 268 (2010) 1818, SRIM software 〈http://www.srim.org $\rangle$.

[17] ORTEC LO-AX: Low energy photon detector 〈http://www.ortec-online.com/ Solutions/gamma-spectroscopy.aspx>.

[18] J.L. Herraiz, et al., IEEE Transactions on Nuclear Science 58 (2011) 2257.

[19] V. Bettinardi, et al., European Journal of Nuclear Medicine and Molecular maging 29 (2002) 7

[20] J. Cal-González, et al., IEEE Transactions Medical Imaging 34 (2015) 2394

[21] S. España, et al., Physics in Medicine and Biology 54 (2009) 1723.

[22] C. Champion, C.L. Loirec, Physics in Medicine and Biology 52 (2007) 6605.

[23] M. Partridge, et al., Nuclear Instruments and Methods in Physics A 568 (2006) 933. 
[24] S.E. Derenzo et al., International Congress Series 1030, in: Proceedings of Brain PET'93, Akita, Japan, May, 29-31, 1993.

[25] Z.H. Cho, et al., Journal of Nuclear Medicine 16 (1975) 1174.

[26] B. Bai, et al., IEEE Nuclear Science Symposium Conference Record (2003) 2501.

[27] M. Hossain, M. Su, Journal of Physical Chemistry C 116 (2012) 23047.
[28] C. Bárcena, et al., Chemical Communications 19 (2008) 2224.

[29] K. Parodi, W. Enghardt, T. Haberer, Physics in Medicine and Biology 47 (2002) 21. 